\title{
Master Surgical Schedule Planning to Reduce Variability in Post-Operative Ward Bed Demand
}

\author{
Rafael Calegari \\ Federal University of Rio Grande do Sul \\ Flavio Fogliatto \\ Federal University of Rio Grande do Sul \\ Filipe Lucini \\ University of Calgary \\ João Brito \\ Federal University of Rio Grande do Sul \\ Gabrielli Yamashita \\ Federal University of Rio Grande do Sul \\ Michel Anzanello \\ Federal University of Rio Grande do Sul \\ Guilherme Tortorella ( $\nabla$ gtortorella@bol.com.br) \\ Universidade Federal de Santa Catarina

\section{Beatriz Schaan} \\ Federal University of Rio Grande do Sul
}

\section{Research Article}

Keywords: OR in health services, master surgical schedule, operating room timetable, ward bed demand variability, genetic algorithm

Posted Date: February 16th, 2022

DOl: https://doi.org/10.21203/rs.3.rs-1319005/v1

License: (c) (i) This work is licensed under a Creative Commons Attribution 4.0 International License. Read Full License 


\section{Abstract}

Peaks in patients' demand for inward hospitalization usually lead to disruptions in the provision of healthcare, having negative effects on patient and staff satisfaction. The two main sources of ward bed demand are the emergency department and the surgical center; while the former is random by nature, the latter may be managed through proper allocation of surgical specialties to time slots (or blocks) in the center's timetable (or Master Surgical Schedule - MSS), and efficient scheduling of surgical procedures within time slots across specialties. We propose a three-step method to design an MSS timetable. In step 1, we mine historical data to determine the average duration of surgical procedures and the average length of stay in wards required by each surgical specialty. In step 2, we use a genetic algorithm to determine a good quality timetable that minimizes the ward bed demand variability overall specialties. In step 3 , we approximate the new timetable to the one currently in use at the hospital through a refinement heuristic. Our propositions were tested using data from a tertiary public teaching hospital. The resulting timetable reduced post-operative ward bed demand variability by $99.9 \%$, keeping $97 \%$ of surgical specialties allocated in their original slots. To the best of our knowledge, this is the first method for long-term MSS design that reduces post-operative ward bed demand variability and changes in allocations in the current surgical center's timetable. We innovate by considering the hospital's current timetable to search for solutions promoting minimum changes to the surgical center's operation.

\section{Full Text}

This preprint is available for download as a PDF.

\section{Tables}

Table 1. List of surgical specialties and associated relevant information 
Specialty,

\begin{tabular}{llllllll}
\hline 1. Pediatrics & PED & 2963 & 1403.36 & 361.1 & 12 & 1.65 & 386.01 \\
\hline 2. Colorectal & CRT & 2516 & 651.46 & 178.6 & 8 & 3.52 & 382.48 \\
\hline 3. Neurosurgery & NEU & 2392 & 637.57 & 137.3 & 10 & 4.05 & 430.79 \\
\hline 4. Digestive System & DIG & 9930 & 528.73 & 141.8 & 23 & 3.4 & 299.73 \\
\hline 5. Urology & URO & 11136 & 526.64 & 136.4 & 25 & 2.63 & 231.17 \\
\hline 6. Vascular & VAS & 3435 & 525.74 & 130.1 & 12 & 3.03 & 265.32 \\
\hline 7. Plastic & PLA & 1663 & 493.35 & 161.7 & 8 & 3.63 & 298.23 \\
\hline 8. Thoracic & THO & 3213 & 458.63 & 88.0 & 10 & 3.48 & 266.07 \\
\hline 9. Private & PRI & 14949 & 419.84 & 98.7 & 48 & 3.22 & 225.76 \\
\hline 10. General & GEN & 9294 & 377.37 & 101.2 & 22 & 3.03 & 190.38 \\
\hline 11. Orthopedics and Traumatology & ORT & 6468 & 312.08 & 71.1 & 22 & 3.18 & 165.37 \\
\hline 12. Gynecology and Obstetrics & OBG & 5398 & 210.51 & 85.6 & 18 & 2.61 & 91.4 \\
\hline 13. Otorhino & OTR & 5652 & 204.33 & 43.6 & 18 & 2.96 & 100.82 \\
\hline 14. Oral and Maxillofacial & OMF & 278 & 178.84 & 23.7 & 2 & 3.9 & 116.36 \\
\hline 15. Mastology & MAS & 2034 & 72.57 & 18.4 & 6 & 2.93 & 35.48 \\
\hline 16. Undefined & Und & 0 & 0 & 0 & 86 & 0 & 0 \\
\hline
\end{tabular}

Table 2. Current surgical center's timetable 
Week 1

Room Shift Mon Tue Wed Thu Fri Mon Tue Wed Thu Fri

$1 \quad M \quad$ PRI $\quad$ PLA THO OMF PLA PRI PLA THO GEN PLA

A PRI PLA THO OMF PLA PRI PLA THO GEN PLA

E PRI PRI

$2 \quad M \quad$ GEN DIG $\quad$ OBG URO GEN DIG OBG URO

$\begin{array}{llll}A & \text { PRI PRI PRI PRI }\end{array}$

$E$

$3 \quad M \quad$ OBG OBG $\mathrm{MAS}$ OBG OBG OBG OBG MAS OBG OBG

$A$ OBG OBG MAS OBG OBG OBG OBG MAS OBG OBG

E

$4 \quad M \quad$ VAS ORT ORT ORT NEU VAS ORT ORT ORT NEU

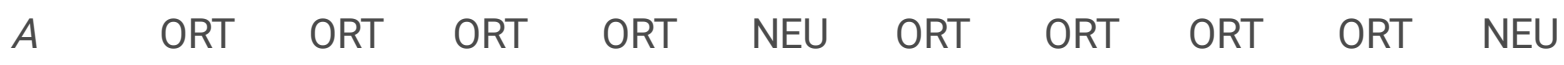

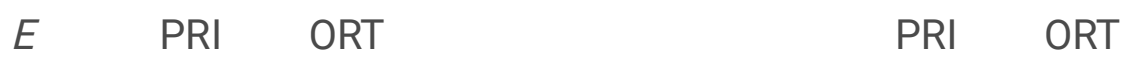

5 D DIG DIG DIG GEN PRI DIG DIG DIG GEN PRI

$A$ DIG DIG GEN GEN PRI DIG DIG GEN GEN PRI

E PRI PRI

6 M PED PRI PED VAS PED PED PRI URO VAS PED

$A$ PED PRI PED DIG PRI PED PRI PED PED PRI

$\begin{array}{lllll}E & \text { PRI PED } & \text { PRI PED }\end{array}$

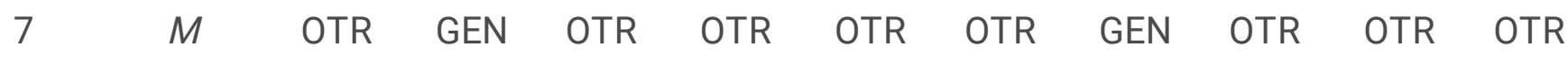
$A$ OTR OTR OTR OTR OTR OTR OTR OTR OTR OTR $E$

$8 \quad M \quad$ URO URO PRI URO URO URO URO PRI URO URO $A$ URO URO PRI PRI URO URO URO PRI PRI URO $\begin{array}{lllllll}E & \text { URO } & \text { PRI } & \text { PRI } & \text { URO } & \text { PRI } & \text { PRI }\end{array}$

$10 M$ ORT VAS DIG GEN URO ORT VAS DIG GEN URO A VAS ORT DIG GEN URO VAS ORT DIG GEN URO

$\begin{array}{llllll}E & \text { URO } & \text { PRI } & \text { DIG } & \text { URO } & \text { PRI }\end{array}$

$11 M \quad$ CRT DIG CRT CRT THO CRT DIG CRT CRT THO 


\begin{tabular}{|c|c|c|c|c|c|c|c|c|c|c|c|}
\hline & $A$ & MAS & DIG & $\mathrm{THO}$ & CRT & THO & MAS & DIG & $\mathrm{THO}$ & CRT & THO \\
\hline & $E$ & & & & & & & & & & \\
\hline \multirow[t]{3}{*}{13} & $M$ & GEN & GEN & NEU & ORT & VAS & GEN & GEN & NEU & ORT & VAS \\
\hline & $A$ & GEN & NEU & PRI & PRI & VAS & GEN & NEU & PRI & PRI & VAS \\
\hline & $E$ & & NEU & PRI & PRI & PRI & & NEU & PRI & PRI & PRI \\
\hline
\end{tabular}

Table 3. Timetable I (T-I) 
Week 1

Room Shift Mon Tue Wed Thu Fri

1

$M \quad$ PR

A

$A \quad \mathrm{PR}$

E

2

$M \quad$ GEN DIG

$\begin{array}{lll}A & \text { PRI } & \text { PRI }\end{array}$

E

3

3

\begin{tabular}{|c|c|c|c|c|c|c|}
\hline & & & & & & Week \\
\hline Shift & Mon & Tue & Wed & Thu & Fri & Mon \\
\hline$M$ & PRI & PLA & $\mathrm{THO}$ & URO* & PLA & PRI \\
\hline$A$ & PRI & OTR* & THO & URO* & MAS* & PRI \\
\hline$E$ & PRI & & & & & PRI \\
\hline$M$ & GEN & DIG & & OBG & URO & GEN \\
\hline$A$ & PRI & PRI & & & & PRI \\
\hline \multicolumn{7}{|l|}{$E$} \\
\hline$M$ & OBG & OBG & MAS & OBG & OBG & OBG \\
\hline$A$ & PRI* & * & OBG* & OBG & OTR* & OBG \\
\hline \multicolumn{7}{|c|}{$E$} \\
\hline$M$ & VAS & ORT & ORT & ORT & NEU & THO* \\
\hline$A$ & ORT & ORT & ORT & ORT & NEU & THO* \\
\hline$E$ & PRI & ORT & & & & PRI \\
\hline$M$ & DIG & DIG & DIG & GEN & PRI & DIG \\
\hline$A$ & VAS* & DIG & GEN & GEN & PRI & DIG \\
\hline$E$ & & & & & PRI & \\
\hline$M$ & NEU* & PRI & PED & VAS & PED & PED \\
\hline$A$ & NEU* & PRI & PLA* & DIG & PRI & DIG* \\
\hline$E$ & & PRI & VAS* & & & \\
\hline$M$ & THO* & OMF* & OTR & OTR & OTR & OTR \\
\hline
\end{tabular}

\begin{tabular}{|c|c|c|c|c|c|c|}
\hline & & & & & & Week \\
\hline Shift & Mon & Tue & Wed & Thu & Fri & Mon \\
\hline$M$ & PRI & PLA & $\mathrm{THO}$ & URO* & PLA & PRI \\
\hline$A$ & PRI & OTR* & THO & URO* & MAS* & PRI \\
\hline$E$ & PRI & & & & & PRI \\
\hline$M$ & GEN & DIG & & OBG & URO & GEN \\
\hline$A$ & PRI & PRI & & & & PRI \\
\hline \multicolumn{7}{|l|}{$E$} \\
\hline$M$ & OBG & OBG & MAS & OBG & OBG & OBG \\
\hline$A$ & PRI* & * & OBG* & OBG & OTR* & OBG \\
\hline \multicolumn{7}{|c|}{$E$} \\
\hline$M$ & VAS & ORT & ORT & ORT & NEU & THO* \\
\hline$A$ & ORT & ORT & ORT & ORT & NEU & THO* \\
\hline$E$ & PRI & ORT & & & & PRI \\
\hline$M$ & DIG & DIG & DIG & GEN & PRI & DIG \\
\hline$A$ & VAS* & DIG & GEN & GEN & PRI & DIG \\
\hline$E$ & & & & & PRI & \\
\hline$M$ & NEU* & PRI & PED & VAS & PED & PED \\
\hline$A$ & NEU* & PRI & PLA* & DIG & PRI & DIG* \\
\hline$E$ & & PRI & VAS* & & & \\
\hline$M$ & THO* & OMF* & OTR & OTR & OTR & OTR \\
\hline
\end{tabular}

E

4

\begin{tabular}{|c|c|c|c|c|c|c|}
\hline & & & & & & Week \\
\hline Shift & Mon & Tue & Wed & Thu & Fri & Mon \\
\hline$M$ & PRI & PLA & $\mathrm{THO}$ & URO* & PLA & PRI \\
\hline$A$ & PRI & OTR* & $\mathrm{THO}$ & URO* & MAS* & PRI \\
\hline$E$ & PRI & & & & & PRI \\
\hline$M$ & GEN & DIG & & OBG & URO & GEN \\
\hline$A$ & PRI & PRI & & & & PRI \\
\hline \multicolumn{7}{|l|}{$E$} \\
\hline$M$ & OBG & OBG & MAS & OBG & OBG & OBG \\
\hline$A$ & PRI* & * & OBG* & OBG & OTR* & OBG \\
\hline \multicolumn{7}{|l|}{$E$} \\
\hline$M$ & VAS & ORT & ORT & ORT & NEU & THO* \\
\hline$A$ & ORT & ORT & ORT & ORT & NEU & THO* \\
\hline$E$ & PRI & ORT & & & & PRI \\
\hline$M$ & DIG & DIG & DIG & GEN & PRI & DIG \\
\hline$A$ & VAS* & DIG & GEN & GEN & PRI & DIG \\
\hline$E$ & & & & & PRI & \\
\hline$M$ & NEU* & PRI & PED & VAS & PED & PED \\
\hline$A$ & NEU* & PRI & PLA* & DIG & PRI & DIG* \\
\hline$E$ & & PRI & VAS* & & & \\
\hline$M$ & THO* & OMF* & OTR & OTR & OTR & OTR \\
\hline
\end{tabular}

$\begin{array}{llll}A & \text { ORT ORT ORT }\end{array}$

$\begin{array}{lll}E & \mathrm{PRI} & \mathrm{ORT}\end{array}$

5

$M$

\begin{tabular}{|c|c|c|c|c|c|c|}
\hline & & & & & & \\
\hline Shift & Mon & Tue & Wed & Thu & Fri & Mon \\
\hline$M$ & PRI & PLA & THO & URO* & PLA & PRI \\
\hline$A$ & PRI & OTR* & THO & URO* & MAS* & PRI \\
\hline$E$ & PRI & & & & & PRI \\
\hline$M$ & GEN & DIG & & OBG & URO & GEN \\
\hline$A$ & PRI & PRI & & & & PRI \\
\hline \multicolumn{7}{|l|}{$E$} \\
\hline$M$ & OBG & OBG & MAS & OBG & OBG & OBG \\
\hline$A$ & PRI* & * & OBG* & OBG & OTR* & OBG \\
\hline \multicolumn{7}{|c|}{$E$} \\
\hline$M$ & VAS & ORT & ORT & ORT & NEU & THO* \\
\hline$A$ & ORT & ORT & ORT & ORT & NEU & THO* \\
\hline$E$ & PRI & ORT & & & & PRI \\
\hline$M$ & DIG & DIG & DIG & GEN & PRI & DIG \\
\hline$A$ & VAS* & DIG & GEN & GEN & PRI & DIG \\
\hline$E$ & & & & & PRI & \\
\hline$M$ & NEU* & PRI & PED & VAS & PED & PED \\
\hline$A$ & NEU* & PRI & $\mathrm{PLA}^{*}$ & DIG & PRI & DIG* \\
\hline$E$ & & PRI & VAS* & & & \\
\hline$M$ & THO* & OMF* & OTR & OTR & OTR & OTR \\
\hline
\end{tabular}

A

6

E

$M \quad$ NEU* PR

\begin{tabular}{|c|c|c|c|c|c|c|}
\hline & & & & & & Week \\
\hline Shift & Mon & Tue & Wed & Thu & Fri & Mon \\
\hline$M$ & PRI & PLA & $\mathrm{THO}$ & URO* & PLA & PRI \\
\hline$A$ & PRI & OTR* & THO & URO* & MAS* & PRI \\
\hline$E$ & PRI & & & & & PRI \\
\hline$M$ & GEN & DIG & & OBG & URO & GEN \\
\hline$A$ & PRI & PRI & & & & PRI \\
\hline \multicolumn{7}{|l|}{$E$} \\
\hline$M$ & OBG & OBG & MAS & OBG & OBG & OBG \\
\hline$A$ & PRI* & * & OBG* & OBG & OTR* & OBG \\
\hline \multicolumn{7}{|c|}{$E$} \\
\hline$M$ & VAS & ORT & ORT & ORT & NEU & THO* \\
\hline$A$ & ORT & ORT & ORT & ORT & NEU & THO* \\
\hline$E$ & PRI & ORT & & & & PRI \\
\hline$M$ & DIG & DIG & DIG & GEN & PRI & DIG \\
\hline$A$ & VAS* & DIG & GEN & GEN & PRI & DIG \\
\hline$E$ & & & & & PRI & \\
\hline$M$ & NEU* & PRI & PED & VAS & PED & PED \\
\hline$A$ & NEU* & PRI & PLA* & DIG & PRI & DIG* \\
\hline$E$ & & PRI & VAS* & & & \\
\hline$M$ & THO* & OMF* & OTR & OTR & OTR & OTR \\
\hline
\end{tabular}

E

7

7

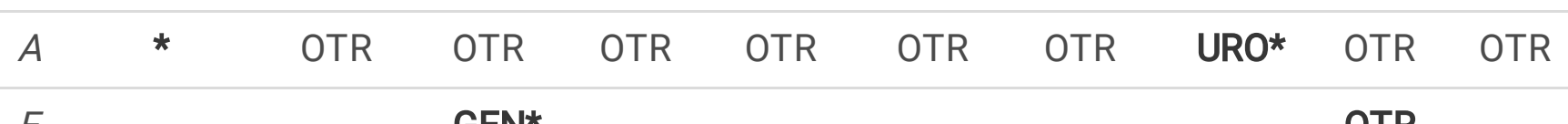

E

8

$M \quad$ URO URO PRI URO URO URO PRI* PRI URO URO

A URO MAS* PRI PRI URO THO* * $\quad$ PRI PRI URO

E URO PRI PRI $\quad$ THO* $\quad$ PRI PRI

$10 \quad M \quad$ ORT VAS URO* GEN ORT* NEU* CRT* DIG PLA URO

A VAS ORT URO* GEN ORT* NEU* * URO* PLA DIG*

E CRT* PRI PRI* DIG ORT* * $\quad$ PRI

11 CRT DIG CRT URO* GEN* PLA* PED* VAS* CRT ORT* 


\begin{tabular}{lllllllllll}
\hline$A$ & MAS & DIG & OBG* & URO* & GEN* & $*$ & PED* & VAS* & CRT & ORT* \\
\hline$E$ & & & OBG* & URO* & GEN* & & & & OMF & PRI* \\
\hline$M$ & GEN & PED* & NEU & DIG* & VAS* & GEN & $*$ & URO* & ORT & VAS \\
\hline A & ORT* & PED* & PRI & DIG* & CRT* & PRI* & NEU & PRI & PRI & VAS \\
\hline$E$ & & $*$ & PRI & PED* & CRT* & & NEU & $*$ & THO & PRI
\end{tabular}

*: Unmatched slots with respect to current timetable

Table 4. Timetable II (T-II) 
Week 1

Room Shift Mon Tue Wed Thu Fri Mon

1 M PRI PLA THO PED* PLA PRI PLA THO GEN PLA

$A$ PRI PLA * OMF PLA PRI PLA THO GEN PLA

E PRI PRI

$2 \quad M \quad$ GEN DIG OBG URO GEN DIG OBG URO

$\begin{array}{llll}A & \text { PRI PRI } & \text { PRI PRI }\end{array}$

E

$3 \quad M \quad$ OBG OBG MAS OBG OBG OBG OBG $M A S$ OBG OBG

$A$ OBG OBG MAS OBG OBG OBG OBG MAS OBG OBG

E

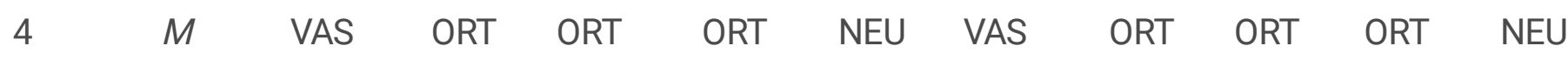

$\begin{array}{lllllllll}A & \text { ORT } & \text { ORT } & \text { ORT } & \text { ORT NEU ORT ORT ORT } & \text { ORT NEU }\end{array}$

$\begin{array}{llll}E & \text { PRI ORT } & \text { PRI } & \text { ORT }\end{array}$

$5 \quad M \quad$ DIG DIG DIG GEN PRI DIG DIG DIG GEN PRI

$A$ DIG DIG GEN GEN PRI DIG DIG GEN GEN PRI

E PRI PRI

6 M ORT* PRI PED VAS PED PED PRI URO VAS PED

$A$ OTR* PRI PED DIG PRI OMF* PRI PED PED PRI

$\begin{array}{llll}E & \text { PRI } & \text { PED } & \text { PRI PED }\end{array}$

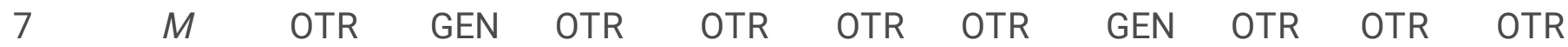

$A$ OTR OTR OTR OTR OTR OTR OTR OTR PED* OTR

$E$

$8 \quad M \quad$ URO URO PRI URO URO URO URO PRI URO URO

$\begin{array}{llllllllll}A & \text { URO URO PRI } & \text { PRI } & \text { URO } & \text { URO } & \text { URO } & \text { PRI } & \text { PRI } & \text { URO }\end{array}$

$\begin{array}{lllllll}E & \text { URO } & \text { PRI } & \text { PRI } & \text { URO } & \text { PRI } & \text { PRI }\end{array}$

$10 M$ ORT VAS DIG GEN URO ORT VAS DIG GEN URO

A VAS ORT DIG GEN URO VAS ORT DIG GEN URO

$\begin{array}{llllll}E & \text { URO PRI } & \text { DIG } & \text { URO } & \text { PRI }\end{array}$

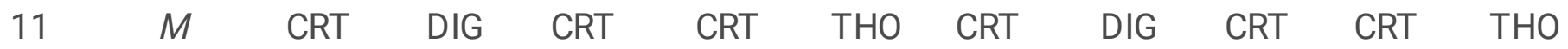




\begin{tabular}{clllllllllll}
\hline & $A$ & THO* & DIG & MAS* & CRT & THO & MAS & DIG & THO & CRT & THO \\
\hline$E$ & & & & & & & & & & \\
\hline 13 & $M$ & GEN & GEN & NEU & PED* & VAS & GEN & GEN & NEU & ORT & VAS \\
\hline & GEN & NEU & PRI & PRI & VAS & GEN & NEU & PRI & PRI & VAS \\
\hline & THO* & NEU & PRI & PRI & PRI & & NEU & PRI & PRI & PRI
\end{tabular}

*: Unmatched slots with respect to current timetable

Table 5. Comparison between CT, T-I and T-II

\section{Current timetable Timetable I Timetable II}

\begin{tabular}{llll}
\hline Average & 11,218 & 11,218 & 11,218 \\
\hline Standard Deviation & 999 & 3.5 & 97.4 \\
\hline Coefficient of Variation & $8.9 \%$ & $0.03 \%$ & $0.9 \%$ \\
\hline Variance & 998,222 & 12.3 & 9,497 \\
\hline Minimum & 8,936 & 11,214 & 10,987 \\
\hline Maximum & 12,431 & 11,224 & 11,407 \\
\hline Range & 3,495 & 10 & 420
\end{tabular}

\section{Figures}

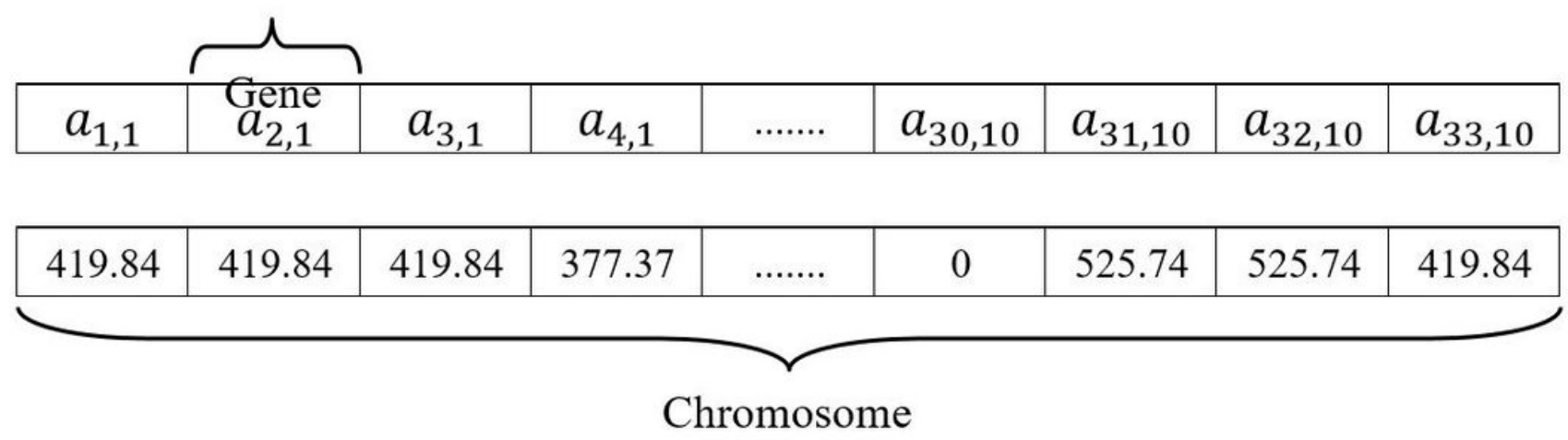

Figure 1

Chromosome enconding using data from the current timetable 


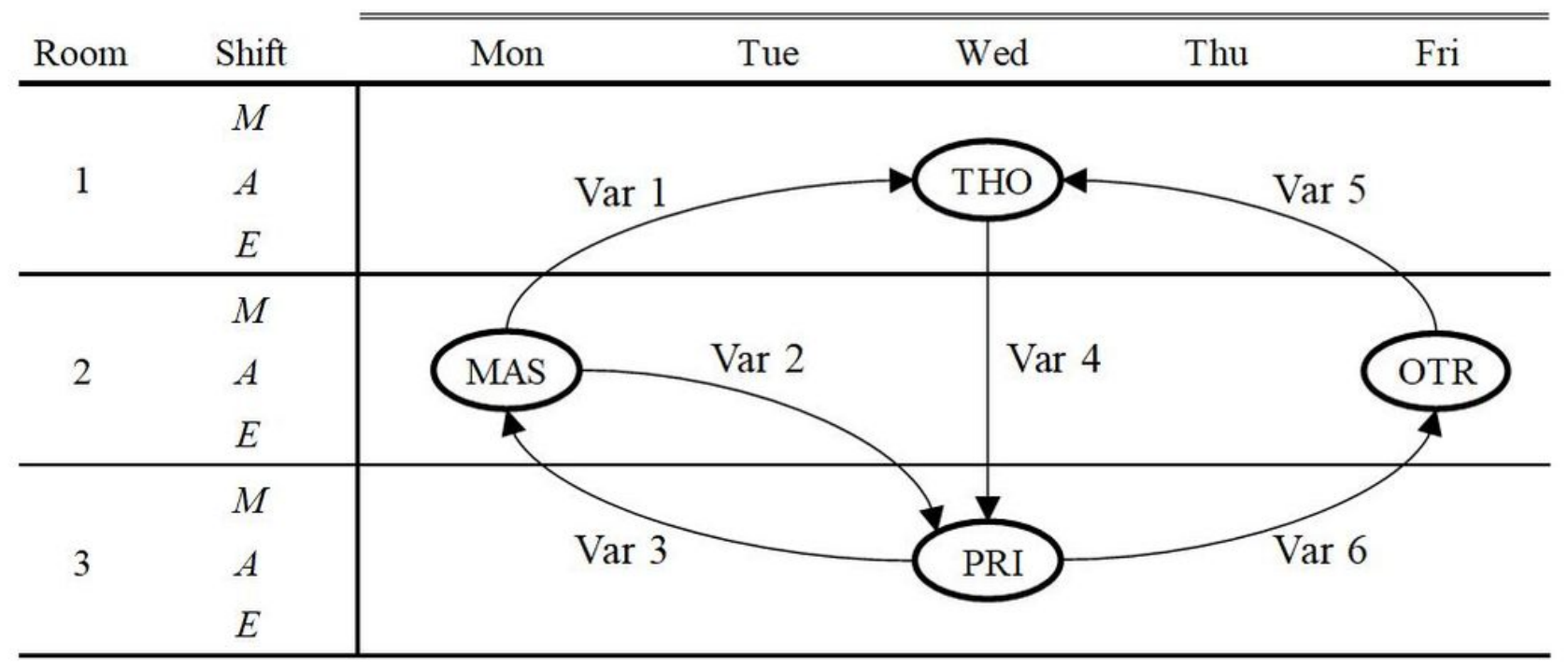

Figure 2

Network depicting a surgical center with 3 ORs working 3 shifts during 5 weekdays

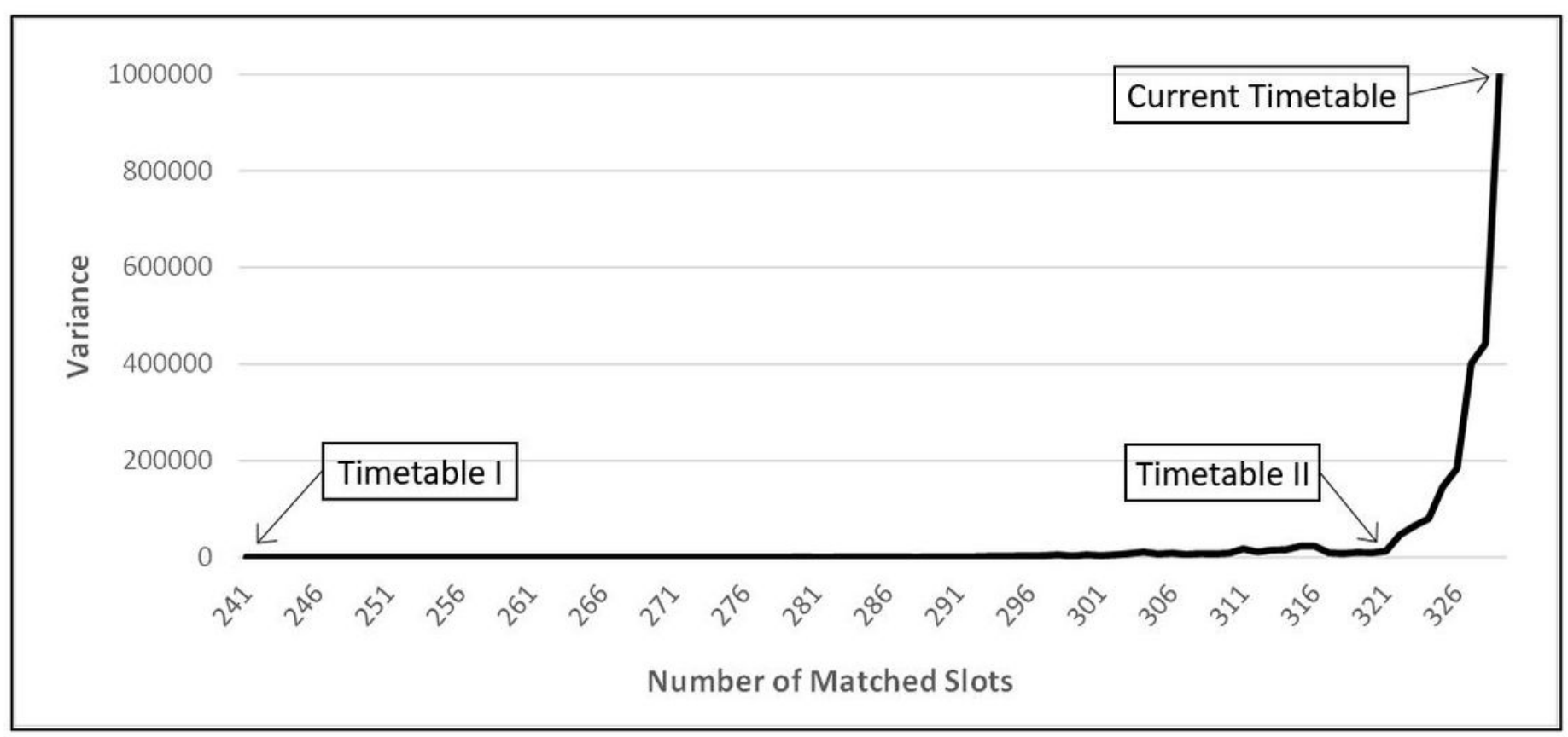

Figure 3

Variance in post-operative demand for bed hours as a function of NMS in the timetable 


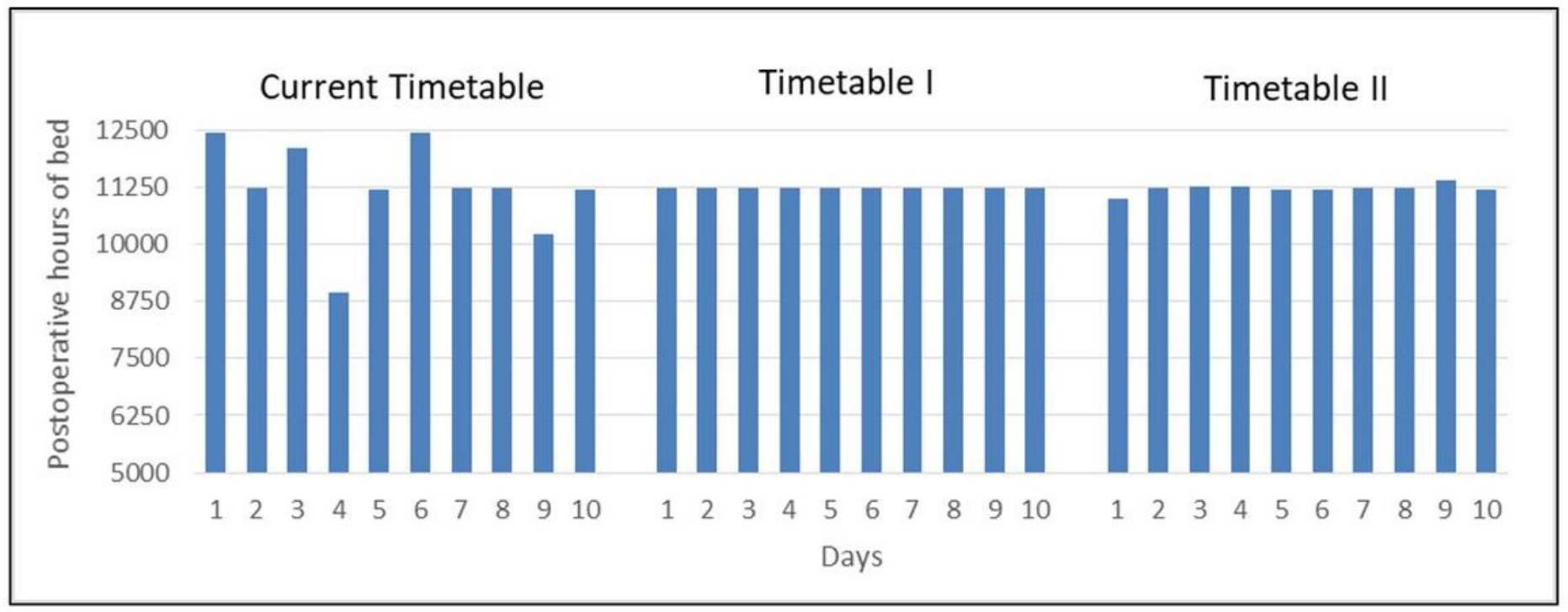

Figure 4

Histogram of daily demand for post-operative bed hours in CT, T-I and T-II

\section{Supplementary Files}

This is a list of supplementary files associated with this preprint. Click to download.

- Appendix.docx 